\title{
ENTROPY OF EIGENFUNCTIONS
}

\author{
NALINI ANANTHARAMAN, HERBERT KOCH, AND STÉPHANE NONNENMACHER
}

\begin{abstract}
We study the high-energy limit for eigenfunctions of the laplacian, on a compact negatively curved manifold. We review the recent result of AnantharamanNonnenmacher [4] giving a lower bound on the Kolmogorov-Sinai entropy of semiclassical measures. The bound proved here improves the result of 4 in the case of variable negative curvature.
\end{abstract}

\section{Motivations}

The theory of quantum chaos tries to understand how the chaotic behaviour of a classical Hamiltonian system is reflected in its quantum counterpart. For instance, let $M$ be a compact Riemannian $C^{\infty}$ manifold, with negative sectional curvatures. The geodesic flow has the Anosov property, which is considered as the ideal chaotic behaviour in the theory of dynamical systems. The corresponding quantum dynamics is the unitary flow generated by the Laplace-Beltrami operator on $L^{2}(M)$. One expects that the chaotic properties of the geodesic flow influence the spectral theory of the Laplacian. The Random Matrix conjecture [7] asserts that the large eigenvalues should, after proper unfolding, statistically resemble those of a large random matrix, at least for a generic Anosov metric. The Quantum Unique Ergodicity conjecture [26] (see also [6, 30]) describes the corresponding eigenfunctions $\psi_{k}$ : it claims that the probability measure $\left|\psi_{k}(x)\right|^{2} d x$ should approach (in the weak topology) the Riemannian volume, when the eigenvalue tends to infinity. In fact a stronger property should hold for the Wigner transform $W_{\psi}$, a function on the cotangent bundle $T^{*} M$, (the classical phase space) which simultaneously describes the localization of the wave function $\psi$ in position and momentum.

We will adopt a semiclassical point of view, that is consider the eigenstates of eigenvalue unity of the semiclassical Laplacian $-\hbar^{2} \triangle$, thereby replacing the high-energy limit by the semiclassical limit $\hbar \rightarrow 0$. We denote by $\left(\psi_{k}\right)_{k \in \mathbb{N}}$ an orthonormal basis of $L^{2}(M)$ made of eigenfunctions of the Laplacian, and by $\left(-\frac{1}{\hbar_{k}^{2}}\right)_{k \in \mathbb{N}}$ the corresponding eigenvalues:

$$
-\hbar_{k}^{2} \triangle \psi_{k}=\psi_{k}, \text { with } \hbar_{k+1} \leq \hbar_{k} .
$$

We are interested in the high-energy eigenfunctions of $-\triangle$, in other words the semiclassical limit $\hbar_{k} \rightarrow 0$.

The Wigner distribution associated to an eigenfunction $\psi_{k}$ is defined by

$$
W_{k}(a)=\left\langle\mathrm{Op}_{\hbar_{k}}(a) \psi_{k}, \psi_{k}\right\rangle_{L^{2}(M)}, \quad a \in C_{c}^{\infty}\left(T^{*} M\right) .
$$

Here $\mathrm{Op}_{\hbar_{k}}$ is a quantization procedure, set at the scale (wavelength) $\hbar_{k}$, which associates to any smooth phase space function $a$ (with nice behaviour at infinity) a bounded operator on 
$L^{2}(M)$. See for instance [13] or [14] for various quantizations $\mathrm{Op}_{\hbar}$ on $\mathbb{R}^{d}$. On a manifold, one can use local coordinates to define Op in a finite system of charts, then glue the objects defined locally thanks to a smooth partition of unity [11]. For standard quantizations $\mathrm{Op}_{\hbar_{k}}$, the Wigner distribution is of the form $W_{k}(x, \xi) d x d \xi$, where $W_{k}(x, \xi)$ is a smooth function on $T^{*} M$, called the Wigner transform of $\psi$. If $a$ is a function on the manifold $M, \mathrm{Op}_{\hbar}(a)$ can be taken as the multiplication by $a$, and thus we have $W_{k}(a)=\int_{M} a(x)\left|\psi_{k}(x)\right|^{2} d x$ : the Wigner transform is thus a microlocal lift of the density $\left|\psi_{k}(x)\right|^{2}$. Although the definition of $W_{k}$ depends on a certain number of choices, like the choice of local coordinates, or of the quantization procedure (Weyl, anti-Wick, "right" or "left" quantization...), its asymptotic behaviour when $\hbar_{k} \longrightarrow 0$ does not. Accordingly, we call semiclassical measures the limit points of the sequence $\left(W_{k}\right)_{k \in \mathbb{N}}$, in the distribution topology.

In the semiclassical limit, "quantum mechanics converges to classical mechanics". We will denote $|\cdot|_{x}$ the norm on $T_{x}^{*} M$ given by the metric. The geodesic flow $\left(g^{t}\right)_{t \in \mathbb{R}}$ is the Hamiltonian flow on $T^{*} M$ generated by the Hamiltonian $H(x, \xi)=\frac{|\xi|_{x}^{2}}{2}$. A quantization of this Hamiltonian is given by the rescaled Laplacian $-\frac{\hbar^{2} \triangle}{2}$, which generates the unitary flow $\left(U_{\hbar}^{t}\right)=\left(\exp \left(i t \hbar \frac{\Delta}{2}\right)\right)$ acting on $L^{2}(M)$. The semiclassical correspondence of the flows $\left(U_{\hbar}^{t}\right)$ and $\left(g^{t}\right)$ is expressed through the Egorov Theorem :

Theorem 1.1. Let $a \in C_{c}^{\infty}\left(T^{*} M\right)$. Then, for any given $t$ in $\mathbb{R}$,

$$
\left\|U_{\hbar}^{-t} \mathrm{Op}_{\hbar}(a) U_{\hbar}^{t}-\mathrm{Op}_{\hbar}\left(a \circ g^{t}\right)\right\|_{L^{2}(M)}=\mathcal{O}(\hbar), \quad \hbar \rightarrow 0 .
$$

The constant implied in the remainder grows (often exponentially) with $t$, which represents a notorious problem when one wants to study the large time behaviour of $\left(U_{\hbar}^{t}\right)$. Typically, the quantum-classical correspondence will break down for times $t$ of the order of the Ehrenfest time (3.25).

Using (1.2) and other standard semiclassical arguments, one shows the following :

Proposition 1.2. Any semiclassical measure is a probability measure carried on the energy layer $\mathcal{E}=H^{-1}\left(\frac{1}{2}\right)$ (which coincides with the unit cotangent bundle $S^{*} M$ ). This measure is invariant under the geodesic flow.

Let us call $\mathfrak{M}$ the set of $g^{t}$-invariant probability measures on $\mathcal{E}$. This set is convex and compact for the weak topology. If the geodesic flow has the Anosov property - for instance if $M$ has negative sectional curvature - that set is very large. The geodesic flow has countably many periodic orbits, each of them carrying an invariant probability measure. There are many other invariant measures, like the equilibrium states obtained by variational principles [19], among them the Liouville measure $\mu_{\text {Liouv }}$, and the measure of maximal entropy. Note that, for all these examples of measures, the geodesic flow acts ergodically, meaning that these examples are extremal points in $\mathfrak{M}$. Our aim is to determine, at least partially, the set $\mathfrak{M}_{s c}$ formed by all possible semiclassical measures. By its definition, $\mathfrak{M}_{s c}$ is a closed subset of $\mathfrak{M}$, in the weak topology.

For manifolds such that the geodesic flow is ergodic with respect to the Liouville measure, it has been known for some time that almost all eigenfunctions become equidistributed over $\mathcal{E}$, in the semiclassical limit. This property is dubbed as Quantum Ergodicity : 
Theorem 1.3. [27, 32, 11] Let $M$ be a compact Riemannian manifold, assume that the action of the geodesic flow on $\mathcal{E}=S^{*} M$ is ergodic with respect to the Liouville measure. Let $\left(\psi_{k}\right)_{k \in \mathbb{N}}$ be an orthonormal basis of $L^{2}(M)$ consisting of eigenfunctions of the Laplacian (1.1), and let $\left(W_{k}\right)$ be the associated Wigner distributions on $T^{*} M$.

Then, there exists a subset $\mathcal{S} \subset \mathbb{N}$ of density 1 , such that

$$
W_{k} \longrightarrow \mu_{\text {Liouv }}, \quad k \rightarrow \infty, k \in \mathcal{S} \text {. }
$$

The question of existence of "exceptional" subsequences of eigenstates with a different behaviour is still open. On a negatively curved manifold, the geodesic flow satisfies the ergodicity assumption, and in fact much stronger properties : mixing, $K$-property, etc. For such manifolds, it has been postulated in the Quantum Unique Ergodicity conjecture [26] that the full sequence of eigenstates becomes semiclassically equidistributed over $\mathcal{E}$ : one can take $\mathcal{S}=\mathbb{N}$ in the limit (1.3). In other words, this conjecture states that there exists a unique semiclassical measure, and $\mathfrak{M}_{s c}=\left\{\mu_{\text {Liouv }}\right\}$.

So far the most precise results on this question were obtained for manifolds $M$ with constant negative curvature and arithmetic properties: see Rudnick-Sarnak [26], Wolpert 31. In that very particular situation, there exists a countable commutative family of self-adjoint operators commuting with the Laplacian : the Hecke operators. One may thus decide to restrict the attention to common bases of eigenfunctions, often called "arithmetic" eigenstates, or Hecke eigenstates. A few years ago, Lindenstrauss [24] proved that any sequence of arithmetic eigenstates become asymptotically equidistributed. If there is some degeneracy in the spectrum of the Laplacian, note that it could be possible that the Quantum Unique Ergodicity conjectured by Rudnick and Sarnak holds for one orthonormal basis but not for another. On such arithmetic manifolds, it is believed that the spectrum of the Laplacian has bounded multiplicity: if this is really the case, then the semiclassical equidistribution easily extends to any sequence of eigenstates.

Nevertheless, one may be less optimistic when extending the Quantum Unique Ergodicity conjecture to more general systems. One of the simplest example of a symplectic Anosov dynamical system is given by linear hyperbolic automorphisms of the 2-torus, e.g. Arnold's "cat map" $\left(\begin{array}{ll}2 & 1 \\ 1 & 1\end{array}\right)$. This system can be quantized into a sequence of $N \times N$ unitary matrices - the propagators, where $N \sim \hbar^{-1}$ [18]. The eigenstates of these matrices satisfy a Quantum Ergodicity theorem similar with Theorem 1.3, meaning that almost all eigenstates become equidistributed on the torus in the semiclassical limit [9]. Besides, one can choose orthonormal eigenbases of the propagators, such that the whole sequence of eigenstates is semiclassically equidistributed [22]. Still, because the spectra of the propagators are highly degenerate, one can also construct sequences of eigenstates with a different limit measure [15], for instance, a semiclassical measure consisting in two ergodic components: half of it is the Liouville measure, while the other half is a Dirac peak on a single (unstable) periodic orbit. It was also shown that this half-localization is maximal for this model [16] : a semiclassical measure cannot have more than half its mass carried by a countable union of periodic orbits. The same type of half-localized eigenstates were constructed by two of the authors for another solvable model, namely the "Walsh quantization" of the 
baker's map on the torus [3]; for that model, there exist ergodic semiclassical measures of purely fractal type (that is, without any Liouville component). Another type of semiclassical measure was recently obtained by Kelmer for quantized hyperbolic automorphisms on higher-dimensional tori [20]: it consists in the Lebesgue measure on some invariant co-isotropic subspace of the torus.

For these Anosov models on tori, the construction of exceptional eigenstates strongly uses nongeneric algebraic properties of the classical and quantized systems, and cannot be generalized to nonlinear systems.

\section{MAin RESUlt.}

In order to understand the set $\mathfrak{M}_{s c}$, we will attempt to compute the Kolmogorov-Sinai entropies of semiclassical measures. We work on a compact Riemannian manifold $M$ of arbitrary dimension, and assume that the geodesic flow has the Anosov property. Actually, our method can without doubt be adapted to more general Anosov Hamiltonian systems.

The Kolmogorov-Sinai entropy, also called metric entropy, of a $\left(g^{t}\right)$-invariant probability measure $\mu$ is a nonnegative number $h_{K S}(\mu)$ that describes, in some sense, the complexity of a $\mu$-typical orbit of the flow. The precise definition will be given later, but for the moment let us just give a few facts. A measure carried on a closed geodesic has vanishing entropy. In constant curvature, the entropy is maximal for the Liouville measure. More generally, for any Anosov flow, the energy layer $\mathcal{E}$ is foliated into unstable manifolds of the flow. An upper bound on the entropy of an invariant probability measure is then provided by the Ruelle inequality:

$$
h_{K S}(\mu) \leq\left|\int_{\mathcal{E}} \log J^{u}(\rho) d \mu(\rho)\right| .
$$

In this inequality, $J^{u}(\rho)$ is the unstable Jacobian of the flow at the point $\rho \in \mathcal{E}$, defined as the Jacobian of the map $g^{-1}$ restricted to the unstable manifold at the point $g^{1} \rho$ (note that the average of $\log J^{u}$ over any invariant measure is negative). The equality holds in (2.1) if and only if $\mu$ is the Liouville measure on $\mathcal{E}$ [23]. If $M$ has dimension $d$ and has constant sectional curvature -1 , the above inequality just reads $h_{K S}(\mu) \leq d-1$.

Finally, an important property of the metric entropy is that it is an affine functional on $\mathfrak{M}$. According to the Birkhoff ergodic theorem, for any $\mu \in \mathfrak{M}$ and for $\mu$-almost every $\rho \in \mathcal{E}$, the weak limit

$$
\mu^{\rho}=\lim _{|t| \longrightarrow \infty} \frac{1}{t} \int_{0}^{t} \delta_{g^{s} \rho} d s
$$

exists, and is an ergodic probability measure. We can then write

$$
\mu=\int_{\mathcal{E}} \mu^{\rho} d \mu(\rho)
$$

which realizes the ergodic decomposition of $\mu$. The affineness of the KS entropy means that

$$
h_{K S}(\mu)=\int_{\mathcal{E}} h_{K S}\left(\mu^{\rho}\right) d \mu(\rho) .
$$


An obvious consequence is the fact that the range of $h_{K S}$ on $\mathfrak{M}$ is an interval $\left[0, h_{\max }\right]$.

In the whole article, we consider a certain subsequence of eigenstates $\left(\psi_{k_{j}}\right)_{j \in \mathbb{N}}$ of the Laplacian, such that the corresponding sequence of Wigner distributions $\left(W_{k_{j}}\right)$ converges to a semiclassical measure $\mu$. In the following, the subsequence $\left(\psi_{k_{j}}\right)_{j \in \mathbb{N}}$ will simply be denoted by $\left(\psi_{\hbar}\right)_{\hbar \rightarrow 0}$, using the slightly abusive notation $\psi_{\hbar}=\psi_{\hbar_{k_{j}}}$ for the eigenstate $\psi_{k_{j}}$. Each eigenstate $\psi_{\hbar}$ thus satisfies

$$
\left(-\hbar^{2} \triangle-1\right) \psi_{\hbar}=0 .
$$

In [2] the first author proved that the entropy of any $\mu \in \mathfrak{M}_{s c}$ is strictly positive. In [4], more explicit lower bounds were obtained. The aim of this paper is to improve the lower bounds of [4] into the following

Theorem 2.1. Let $\mu$ be a semiclassical measure associated to the eigenfunctions of the Laplacian on $M$. Then its metric entropy satisfies

$$
h_{K S}(\mu) \geq\left|\int_{\mathcal{E}} \log J^{u}(\rho) d \mu(\rho)\right|-\frac{(d-1)}{2} \lambda_{\max },
$$

where $d=\operatorname{dim} M$ and $\lambda_{\max }=\lim _{t \rightarrow \pm \infty} \frac{1}{t} \log \sup _{\rho \in \mathcal{E}}\left|d g_{\rho}^{t}\right|$ is the maximal expansion rate of the geodesic flow on $\mathcal{E}$.

In particular, if $M$ has constant sectional curvature -1, we have

$$
h_{K S}(\mu) \geq \frac{d-1}{2} .
$$

In dimension $d$, we always have

$$
\left|\int_{\mathcal{E}} \log J^{u}(\rho) d \mu(\rho)\right| \leq(d-1) \lambda_{\max },
$$

so the above bound is an improvement over the one obtained in [4],

$$
h_{K S}(\mu) \geq \frac{3}{2}\left|\int_{\mathcal{E}} \log J^{u}(\rho) d \mu(\rho)\right|-(d-1) \lambda_{\max } .
$$

In the case of constant or little-varying curvature, the bound (2.4) is much sharper than the one proved in [2]. On the other hand, if the curvature varies a lot (still being negative everywhere), the right hand side of (2.3) may actually be negative, in which case the bound is trivial. We believe this "problem" to be a technical shortcoming of our method, and actually conjecture the following bound:

$$
h_{K S}(\mu) \geq \frac{1}{2}\left|\int_{\mathcal{E}} \log J^{u}(\rho) d \mu(\rho)\right| .
$$

Extended to the case of the quantized torus automorphisms or the Walsh-quantized baker's map, this bound is saturated for the half-localized semiclassical measures constructed in [15], as well as those obtained in [20, 3]. This bound allows certain ergodic components to be carried by closed geodesics, as long as other components have positive entropy. This may be compared with the following result obtained by Bourgain and Lindenstrauss in the case of arithmetic surfaces : 
Theorem 2.2. 8] Let $M$ be a congruence arithmetic surface, and $\left(\psi_{j}\right)$ an orthonormal basis of eigenfunctions for the Laplacian and the Hecke operators.

Let $\mu$ be a corresponding semiclassical measure, with ergodic decomposition $\mu=\int_{\mathcal{E}} \mu^{\rho} d \mu(\rho)$. Then, for $\mu$-almost all ergodic components we have $h_{K S}\left(\mu^{\rho}\right) \geq \frac{1}{9}$.

As discussed above, the Liouville measure is the only one satisfying $h_{K S}(\mu)=\left|\int_{\mathcal{E}} \log J^{u}(\rho) d \mu(\rho)\right|$ [23], so the Quantum Unique Ergodicity would be proven in one could replace $1 / 2$ by 1 on the right hand side of (2.6). However, we believe that (2.6) is the optimal result that can be obtained without using much more precise information, like for instance a sharp control on the spectral degeneracies, or fine information on the lengths of closed geodesics.

Indeed, in the above mentioned examples of Anosov systems where the Quantum Unique Ergodicity conjecture is wrong and the bound (2.6) sharp, the quantum spectrum has very high degeneracies, which could be responsible for the possibility to construct exceptional eigenstates. Such high degeneracies are not expected in the case of the Laplacian on a negatively curved manifold. For the moment, however, there is no clear understanding of the precise relation between spectral degeneracies and failure of Quantum Unique Ergodicity.

Acknowledgements. N.A and S.N. were partially supported by the Agence Nationale de la Recherche, under the grant ANR-05-JCJC-0107-01. They benefited from numerous discussions with Y. Colin de Verdière and M. Zworski. S.N. is grateful to the Mathematical Department in Bonn for its hospitality in December 2006.

\section{Outline of the PRoOF}

We start by recalling the definition and some properties of the metric entropy associated with a probability measure on $T^{*} M$, invariant through the geodesic flow. In $§ 3.2$ we extend the notion of entropy to the quantum framework. Our approach is semiclassical, so we want the classical and quantum entropies to be connected in some way when $\hbar \rightarrow 0$. The weights appearing in our quantum entropy are estimated in Thm. 3.1, which was proven and used in [2. In $\$ 3.2 .1$ we also compare our quantum entropy with several "quantum dynamical entropies" previously defined in the literature. The proof of Thm. 2.1 actually starts in $\$ 3.3$, where we present the algebraic tool allowing us to take advantage of our estimates (3.9) (or their optimized version given in Thm. 3.5), namely an "entropic uncertainty principle" specific of the quantum framework. From $\$ 3.4$ on, we apply this "principle" to the quantum entropies appearing in our problem, and proceed to prove Thm. 2.1. Although the method is basically the same as in [4], several small modifications allow to finally obtain the improved lower bound (2.3), and also simplify some intermediate proofs, as explained in Remark 3.6.

3.1. Definition of the metric entropy. In this paper we will meet several types of entropies, all of which are defined using the function $\eta(s)=-s \log s$, for $s \in[0,1]$. We start with the Kolmogorov-Sinai entropy of the geodesic flow with respect to an invariant probability measure.

Let $\mu$ be a probability measure on the cotangent bundle $T^{*} M$. Let $\mathcal{P}=\left(E_{1}, \ldots, E_{K}\right)$ be a finite measurable partition of $T^{*} M: T^{*} M=\bigsqcup_{i=1}^{K} E_{i}$. We will denote the set of indices 
$\{1, \ldots, K\}=\llbracket 1, K \rrbracket$. The Shannon entropy of $\mu$ with respect to the partition $\mathcal{P}$ is defined as

$$
h_{\mathcal{P}}(\mu)=-\sum_{k=1}^{K} \mu\left(E_{k}\right) \log \mu\left(E_{k}\right)=\sum_{k=1}^{K} \eta\left(\mu\left(E_{k}\right)\right) .
$$

For any integer $n \geq 1$, we denote by $\mathcal{P}^{\vee n}$ the partition formed by the sets

$$
E_{\boldsymbol{\alpha}}=E_{\alpha_{0}} \cap g^{-1} E_{\alpha_{1}} \ldots \cap g^{-n+1} E_{\alpha_{n-1}},
$$

where $\boldsymbol{\alpha}=\left(\alpha_{0}, \ldots, \alpha_{n-1}\right)$ can be any sequence in $\llbracket 1, K \rrbracket^{n}$ (such a sequence is said to be of length $|\boldsymbol{\alpha}|=n)$. The partition $\mathcal{P}^{\vee n}$ is called the $n$-th refinement of the initial partition $\mathcal{P}=\mathcal{P}^{\vee 1}$. The entropy of $\mu$ with respect to $\mathcal{P}^{\vee n}$ is denoted by

$$
h_{n}(\mu, \mathcal{P})=h_{\mathcal{P} \vee n}(\mu)=\sum_{\boldsymbol{\alpha} \in[1, K]^{n}} \eta\left(\mu\left(E_{\boldsymbol{\alpha}}\right)\right) .
$$

If $\mu$ is $\left(g^{t}\right)$-invariant, it follows from the convexity of the logarithm that

$$
\forall n, m \geq 1, \quad h_{n+m}(\mu, \mathcal{P}) \leq h_{n}(\mu, \mathcal{P})+h_{m}(\mu, \mathcal{P}),
$$

in other words the sequence $\left(h_{n}(\mu, \mathcal{P})\right)_{n \in \mathbb{N}}$ is subadditive. The entropy of $\mu$ with respect to the action of the geodesic flow and to the partition $\mathcal{P}$ is defined by

$$
h_{K S}(\mu, \mathcal{P})=\lim _{n \rightarrow+\infty} \frac{h_{n}(\mu, \mathcal{P})}{n}=\inf _{n \in \mathbb{N}} \frac{h_{n}(\mu, \mathcal{P})}{n} .
$$

Each weight $\mu\left(E_{\boldsymbol{\alpha}}\right)$ measures the $\mu$-probability to visit successively $E_{\alpha_{0}}, E_{\alpha_{1}}, \ldots, E_{\alpha_{n-1}}$ at times $0,1, \ldots, n-1$ through the geodesic flow. Roughly speaking, the entropy measures the exponential decay of these probabilities when $n$ gets large. It is easy to see that $h_{K S}(\mu, \mathcal{P}) \geq \beta$ if there exists $C$ such that $\mu\left(E_{\boldsymbol{\alpha}}\right) \leq C e^{-\beta n}$, for all $n$ and all $\boldsymbol{\alpha} \in \llbracket 1, K \rrbracket^{n}$.

Finally, the Kolmogorov-Sinai entropy of $\mu$ with respect to the action of the geodesic flow is defined as

$$
h_{K S}(\mu)=\sup _{\mathcal{P}} h_{K S}(\mu, \mathcal{P}),
$$

the supremum running over all finite measurable partitions $\mathcal{P}$. The choice to consider the time 1 of the geodesic flow in the definition (3.1) may seem arbitrary, but the entropy has a natural scaling property : the entropy of $\mu$ with respect to the flow $\left(g^{a t}\right)$ is $|a|$-times its entropy with respect to $\left(g^{t}\right)$.

Assume $\mu$ is carried on the energy layer $\mathcal{E}$. Due to the Anosov property of the geodesic flow on $\mathcal{E}$, it is known that the supremum (3.5) is reached as soon as the diameter of the partition $\mathcal{P} \cap \mathcal{E}$ (that is, the maximum diameter of its elements $E_{k} \cap \mathcal{E}$ ) is small enough. Furthermore, let us assume (without loss of generality) that the injectivity radius of $M$ is larger than 1 . Then, we may restrict our attention to partitions $\mathcal{P}$ obtained by lifting on $\mathcal{E}$ a partition of the manifold $M$, that is take $M=\bigsqcup_{k=1}^{K} M_{k}$ and then $E_{k}=T^{*} M_{k}$. In fact, if the diameter of $M_{k}$ in $M$ is of order $\varepsilon$, then the diameter of the partition $\mathcal{P}^{\vee 2} \cap \mathcal{E}$ in $\mathcal{E}$ is also of order $\varepsilon$. This special choice of our partition is not crucial, but it simplifies certain aspects of the analysis. 
The existence of the limit in (3.4), and the fact that it coincides with the infimum, follow from a standard subadditivity argument. It has a crucial consequence : if $\left(\mu_{i}\right)$ is a sequence of $\left(g^{t}\right)$-invariant probability measures on $T^{*} M$, weakly converging to a probability $\mu$, and if $\mu$ does not charge the boundary of the partition $\mathcal{P}$, we have

$$
h_{K S}(\mu, \mathcal{P}) \geq \limsup _{i} h_{K S}\left(\mu_{i}, \mathcal{P}\right) .
$$

In particular, assume that for $i$ large enough, the following estimates hold :

$$
\forall n \geq 1, \forall \boldsymbol{\alpha} \in \llbracket 1, K \rrbracket^{n}, \quad \mu_{i}\left(E_{\boldsymbol{\alpha}}\right) \leq C_{i} \mathrm{e}^{-\beta n},
$$

with $\beta$ independent of $i$. This implies for $i$ large enough $h_{K S}\left(\mu_{i}, \mathcal{P}\right) \geq \beta$, and this estimate goes to the limit to yield $h_{K S}(\mu) \geq \beta$.

3.2. From classical to quantum dynamical entropy. Since our semiclassical measure $\mu$ is defined as a limit of Wigner distributions $W_{\hbar}$, a naive idea would be to estimate from below the KS entropy of $W_{\hbar}$ and then take the limit $\hbar \rightarrow 0$. This idea cannot work directly, because the Wigner transforms $W_{\hbar}$ are neither positive, nor are they $\left(g^{t}\right)$-invariant. Therefore, one cannot directly use the (formal) integrals $W_{\hbar}\left(E_{\boldsymbol{\alpha}}\right)=\int_{E_{\boldsymbol{\alpha}}} W_{\hbar}(x, \xi) d x d \xi$ to compute the entropy of the semiclassical measure.

Instead, the method initiated by the first author in [2] is based on the following remarks. Each integral $W_{\hbar}\left(E_{\boldsymbol{\alpha}}\right)$ can also be written as $W_{\hbar}\left(\mathbb{1}_{E_{\boldsymbol{\alpha}}}\right)=\int_{T^{*} M} W_{\hbar} \mathbb{1}_{E_{\boldsymbol{\alpha}}}$, where $\mathbb{1}_{E_{\boldsymbol{\alpha}}}$ is the characteristic function on the set $E_{\boldsymbol{\alpha}}$, that is

$$
\mathbb{1}_{E_{\boldsymbol{\alpha}}}=\left(\mathbb{1}_{E_{\alpha_{n-1}}} \circ g^{n-1}\right) \times \ldots \times\left(\mathbb{1}_{E_{\alpha_{1}}} \circ g\right) \times \mathbb{1}_{E_{\alpha_{0}}} .
$$

Remember we took $E_{k}=T^{*} M_{k}$, where the $M_{k}$ form a partition of $M$.

From the definition of the Wigner distribution, this integral corresponds formally to the overlap $\left\langle\psi_{\hbar}, \mathrm{Op}_{\hbar}\left(\mathbb{1}_{E_{\boldsymbol{\alpha}}}\right) \psi_{\hbar}\right\rangle$. Yet, the characteristic functions $\mathbb{1}_{E_{\boldsymbol{\alpha}}}$ have sharp discontinuities, so their quantizations cannot be incorporated in a nice pseudodifferential calculus. Besides, the set $E_{\boldsymbol{\alpha}}$ is not compactly supported, and shrinks in the unstable direction when $n=$ $|\boldsymbol{\alpha}| \longrightarrow+\infty$, so that the operator $\mathrm{Op}_{\hbar}\left(\mathbb{1}_{E_{\boldsymbol{\alpha}}}\right)$ is very problematic.

We also note that an overlap of the form $\left\langle\psi_{\hbar}, \mathrm{Op}_{\hbar}\left(\mathbb{1}_{E_{\boldsymbol{\alpha}}}\right) \psi_{\hbar}\right\rangle$ is a hybrid expression: this is a quantum matrix element of an operator defined in terms of the classical evolution (3.7). From the point of view of quantum mechanics, it is more natural to consider, instead, the operator obtained as the product of Heisenberg-evolved quantized functions, namely

$$
\left(U_{\hbar}^{-n+1} P_{\alpha_{n-1}} U_{\hbar}^{n-1}\right)\left(U_{\hbar}^{-n+2} P_{\alpha_{n-2}} U_{\hbar}^{n-2}\right) \cdots\left(U_{\hbar}^{-1} P_{\alpha_{1}} U_{\hbar}\right) P_{\alpha_{0}} .
$$

Here we used the shorthand notation $P_{k}=\mathbb{1}_{M_{k}}, k \in \llbracket 1, K \rrbracket$ (multiplication operators). To remedy the fact that the functions $\mathbb{1}_{M_{k}}$ are not smooth, which would prevent us from using a semiclassical calculus, we apply a convolution kernel to smooth them, obtain functions $\mathbb{1}_{M_{k}}^{s m} \in C^{\infty}(M)$, and consider $P_{k} \stackrel{\text { def }}{=} \mathbb{1}_{M_{k}}^{s m}$ (we can do this keeping the property $\sum_{k=1}^{K} \mathbb{1}_{M_{k}}^{s m}=$ $1)$.

In the following, we will use the notation $A(t) \stackrel{\text { def }}{=} U_{\hbar}^{-t} A U_{\hbar}^{t}$ for the Heisenberg evolution of the operator $A$ though the Schrödinger flow $U_{\hbar}^{t}=\exp \left(-i t \hbar \frac{\triangle}{2}\right)$. The norm $\|\bullet\|$ will denote 
either the Hilbert norm on $L^{2}(M)$, or the corresponding operator norm. The subsequent "purely quantum" norms were estimated in [2, Thm. 1.3.3]:

Theorem 3.1. (The main estimate [2]) Set as above $P_{k} \stackrel{\text { def }}{=} \mathbb{1}_{M_{k}}^{\text {sm }}$. For every $\mathcal{K}>0$, there exists $\hbar_{\mathcal{K}}>0$ such that, uniformly for all $\hbar<\hbar_{\mathcal{K}}$, for all $n \leq \mathcal{K}|\log \hbar|$, for all $\left(\alpha_{0}, \ldots, \alpha_{n-1}\right) \in \llbracket 1, K \rrbracket^{n}$,

$$
\left\|P_{\alpha_{n-1}}(n-1) P_{\alpha_{n-2}}(n-2) \cdots P_{\alpha_{0}} \psi_{\hbar}\right\| \leq 2(2 \pi \hbar)^{-d / 2} \mathrm{e}^{-\frac{\Lambda}{2} n}(1+\mathcal{O}(\varepsilon))^{n} .
$$

The exponent $\Lambda$ is given by the "smallest expansion rate":

$$
\Lambda=-\sup _{\nu \in \mathfrak{M}} \int \log J^{u}(\rho) d \nu(\rho)=\inf _{\gamma} \sum_{i=1}^{d-1} \lambda_{i}^{+}(\gamma) .
$$

The infimum on the right hand side runs over the set of closed orbits on $\mathcal{E}$, and the $\lambda_{i}^{+}$denote the positive Lyapunov exponents along the orbit, that is the logarithms of the expanding eigenvalues of the Poincaré map, divided by the period of the orbit. The parameter $\varepsilon>0$ is an upper bound on the diameters of the supports of the functions $\mathbb{1}_{M_{k}}^{s m}$ in $M$.

From now on we will call the product operator

$$
P_{\boldsymbol{\alpha}}=P_{\alpha_{n-1}}(n-1) P_{\alpha_{n-2}}(n-2) \cdots P_{\alpha_{0}}, \quad \boldsymbol{\alpha} \in \llbracket 1, K \rrbracket^{n} .
$$

To prove the above estimate, one actually controls the operator norm

$$
\left\|P_{\boldsymbol{\alpha}} \mathrm{Op}_{\hbar}(\chi)\right\| \leq 2(2 \pi \hbar)^{-d / 2} \mathrm{e}^{-\frac{\Lambda}{2} n}(1+\mathcal{O}(\varepsilon))^{n},
$$

where $\chi \in C_{c}^{\infty}\left(\mathcal{E}^{\varepsilon}\right)$ is an energy cutoff such that $\chi=1$ near $\mathcal{E}$, supported inside a neighbourhood $\mathcal{E}^{\varepsilon}=H^{-1}\left(\left[\frac{1}{2}-\varepsilon, \frac{1}{2}+\varepsilon\right]\right)$ of $\mathcal{E}$.

In quantum mechanics, the matrix element $\left\langle\psi_{\hbar}, P_{\boldsymbol{\alpha}} \psi_{\hbar}\right\rangle$ looks like the "probability", for a particle in the state $\psi_{\hbar}$, to visit successively the phase space regions $E_{\alpha_{0}}, E_{\alpha_{1}}, \ldots, E_{\alpha_{n-1}}$ at times $0,1, \ldots, n-1$ of the Schrödinger flow. Theorem 3.1 implies that this "probability" decays exponentially fast with $n$, with rate $\frac{\Lambda}{2}$, but this decay only starts around the time

$$
n_{1} \stackrel{\text { def }}{=} \frac{d|\log \hbar|}{\Lambda}
$$

which is a kind of "Ehrenfest time" (see (3.25) for another definition of Ehrenfest time).

Yet, because the matrix elements $\left\langle\psi_{\hbar}, P_{\boldsymbol{\alpha}} \psi_{\hbar}\right\rangle$ are not real in general, they can hardly be used to define a "quantum measure". Another possibility to define the probability for the particle to visit the sets $E_{\alpha_{k}}$ at times $k$, is to take the squares of the norms appearing in (3.9):

$$
\left\|P_{\boldsymbol{\alpha}} \psi_{\hbar}\right\|^{2}=\left\|P_{\alpha_{n-1}}(n-1) P_{\alpha_{n-2}}(n-2) \cdots P_{\alpha_{0}} \psi_{\hbar}\right\|^{2} .
$$

Now we require the smoothed characteristic functions $\mathbb{1}_{M_{i}}^{s m}$ to satisfy the identity

$$
\sum_{k=1}^{K}\left(\mathbb{1}_{M_{k}}^{s m}(x)\right)^{2}=1 \quad \text { for any point } x \in M
$$


We denote by $\mathcal{P}_{s m}$ the smooth partition of $M$ made by the functions $\left(\left(\mathbb{1}_{M_{k}}^{s m}\right)^{2}\right)_{k=1}^{K}$. The corresponding set of multiplication operators $\left(P_{k}\right)_{k=1}^{K} \stackrel{\text { def }}{=} \mathcal{P}_{q}$ forms a "quantum partition of unity" :

$$
\sum_{k=1}^{K} P_{k}^{2}=I d_{L^{2}}
$$

For any $n \geq 1$, we refine the quantum partition $\mathcal{P}_{q}$ into $\left(P_{\boldsymbol{\alpha}}\right)_{|\boldsymbol{\alpha}|}$, as in (3.10). The weights (3.13) exactly add up to unity, so it makes sense to consider the entropy

$$
h_{n}\left(\psi_{\hbar}, \mathcal{P}_{q}\right) \stackrel{\text { def }}{=} \sum_{\boldsymbol{\alpha} \in[1, K]^{n}} \eta\left(\left\|P_{\boldsymbol{\alpha}} \psi_{\hbar}\right\|^{2}\right) .
$$

3.2.1. Connection with other quantum entropies. This entropy appears to be a particular case of the "general quantum entropies" described by Słomczyński and Życzkowski [28], who already had in mind applications to quantum chaos. In their terminology, a family of bounded operators $\pi=\left(\pi_{k}\right)_{k=1}^{\mathcal{N}}$ on a Hilbert space $\mathcal{H}$ satisfying

$$
\sum_{k=1}^{\mathcal{N}} \pi_{k}^{*} \pi_{k}=I d_{\mathcal{H}}
$$

provides an "instrument" which, to each index $k \in \llbracket 1, \mathcal{N} \rrbracket$, associates the following map on density matrices:

$$
\rho \mapsto \mathcal{I}(k) \rho=\pi_{k} \rho \pi_{k}^{*}, \quad \text { a nonnegative operator with } \operatorname{tr}(\mathcal{I}(k) \rho) \leq 1 .
$$

From a unitary propagator $U$ and its adjoint action $\mathcal{U} \rho=U \rho U^{-1}$, they propose to construct the refined instrument

$$
\mathcal{I}(\boldsymbol{\alpha}) \rho \stackrel{\text { def }}{=} \mathcal{I}\left(\alpha_{n-1}\right) \circ \cdots \mathcal{U} \circ \mathcal{I}\left(\alpha_{1}\right) \circ \mathcal{U} \circ \mathcal{I}\left(\alpha_{0}\right) \rho=U^{-n+1} \pi_{\boldsymbol{\alpha}} \rho \pi_{\boldsymbol{\alpha}}^{*} U^{n-1}, \quad \boldsymbol{\alpha} \in \llbracket 1, \mathcal{N} \rrbracket^{n},
$$

where we used (3.10) to refine the operators $\pi_{k}$ into $\pi_{\boldsymbol{\alpha}}$. We obtain the probability weights

$$
\operatorname{tr}(\mathcal{I}(\boldsymbol{\alpha}) \rho)=\operatorname{tr}\left(\pi_{\boldsymbol{\alpha}} \rho \pi_{\boldsymbol{\alpha}}^{*}\right), \quad \boldsymbol{\alpha} \in \llbracket 1, \mathcal{N} \rrbracket^{n} .
$$

For any $U$-invariant density $\rho$, these weights provide an entropy

$$
h_{n}(\rho, \mathcal{I})=\sum_{\boldsymbol{\alpha} \in[1, \mathcal{N}]^{n}} \eta(\operatorname{tr}(\mathcal{I}(\boldsymbol{\alpha}) \rho)) \text {. }
$$

One easily checks that our quantum partition $\mathcal{P}_{q}=\left(P_{k}\right)_{k=1}^{K}$ satisfies (3.17), and that if one takes $\rho=\left|\psi_{\hbar}\right\rangle\left\langle\psi_{\hbar}\right|$ the weights $\operatorname{tr}(\mathcal{I}(\boldsymbol{\alpha}) \rho)$ exactly correspond to our weights $\left\|P_{\boldsymbol{\alpha}} \psi\right\|^{2}$. Hence, the entropy (3.19) coincides with (3.16).

Around the same time, Alicki and Fannes [1] used the same quantum partition (3.17) (which they called "finite operational partitions of unity") to define a different type of entropy, now called the "Alicki-Fannes entropy" (the definition extends to general $C^{*}$ dynamical systems). For each $n \geq 1$ they extend the weights (3.18) to "off-diagonal entries" to form a $\mathcal{N}^{n} \times \mathcal{N}^{n}$ density matrix $\rho_{n}$ :

$$
\left[\rho_{n}\right]_{\boldsymbol{\alpha}^{\prime}, \boldsymbol{\alpha}}=\operatorname{tr}\left(\pi_{\boldsymbol{\alpha}^{\prime}} \rho \pi_{\boldsymbol{\alpha}}^{*}\right), \quad \boldsymbol{\alpha}, \boldsymbol{\alpha}^{\prime} \in \llbracket 1, \mathcal{N} \rrbracket^{n} .
$$


The AF entropy of the system $(\mathcal{U}, \rho)$ is then defined as follows: take the Von Neumann entropy of these density matrices, $h_{n}^{A F}(\rho, \pi)=\operatorname{tr} \eta\left(\rho_{n}\right)$, then take $\lim \sup _{n \rightarrow \infty} \frac{1}{n} h_{n}^{A F}(\rho, \pi)$ and finally take the supremum over all possible finite operational partitions of unity $\pi$.

We mention that traces of the form (3.20) also appear in the "quantum histories" approach to quantum mechanics (see e.g. [17], and [28, Appendix D] for references).

3.2.2. Naive treatment of the entropy $h_{n}\left(\psi_{\hbar}, \mathcal{P}_{q}\right)$. For fixed $|\boldsymbol{\alpha}|>0$, the Egorov theorem shows that $\left\|P_{\boldsymbol{\alpha}} \psi_{\hbar}\right\|^{2}$ converges to the classical weight $\mu\left(\left(\mathbb{1}_{M_{\boldsymbol{\alpha}}}^{s m}\right)^{2}\right)$ when $\hbar \rightarrow 0$, so for fixed $n>0$ the entropy $h_{n}\left(\psi_{\hbar}, \mathcal{P}_{q}\right)$ converges to $h_{n}\left(\mu, \mathcal{P}_{s m}\right)$, defined as in (3.2), the characteristic functions $\mathbb{1}_{M_{k}}$ being replaced by their smoothed versions $\left(\mathbb{1}_{M_{k}}^{s m}\right)^{2}$. On the other hand, from the estimate (3.11) the entropies $h_{n}\left(\psi_{\hbar}, \mathcal{P}_{q}\right)$ satisfy, for $\hbar$ small enough,

$$
h_{n}\left(\psi_{\hbar}, \mathcal{P}_{q}\right) \geq n(\Lambda+\mathcal{O}(\varepsilon))-d|\log \hbar|+\mathcal{O}(1)
$$

for any time $n \leq \mathcal{K}|\log \hbar|$. For large times $n \approx \mathcal{K}|\log \hbar|$, this provides a lower bound

$$
\frac{1}{n} h_{n}\left(\psi_{\hbar}, \mathcal{P}_{q}\right) \geq(\Lambda+\mathcal{O}(\varepsilon))-\frac{d}{\mathcal{K}}+\mathcal{O}(1 /|\log \hbar|),
$$

which looks very promising since $\mathcal{K}$ can be taken arbitrary large: we could be tempted to take the semiclassical limit, and deduce a lower bound $h_{K S}(\mu) \geq \Lambda$.

Unfortunately, this does not work, because in the range $\left\{n>n_{1}\right\}$ where the estimate (3.21) is useful, the Egorov theorem breaks down, the weights (3.13) do not approximate the classical weights $\mu\left(\left(\mathbb{1}_{M_{\alpha}}^{s m}\right)^{2}\right)$, and there is no relationship between $h_{n}\left(\psi, \mathcal{P}_{q}\right)$ and the classical entropies $h_{n}\left(\mu, \mathcal{P}_{s m}\right)$.

This breakdown of the quantum-classical correspondence around the Ehrenfest time is ubiquitous for chaotic dynamics. It has been observed before when studying the connection between the Alicki-Fannes entropy for the quantized torus automorphisms and the KS entropy of the classical dynamics [5]: the quantum entropies $h_{n}^{A F}\left(\psi_{\hbar}, \mathcal{P}_{q}\right)$ follow the classical $h_{n}\left(\mu, \mathcal{P}_{s m}\right)$ until the Ehrenfest time (and therefore grow linearly with $n$ ), after which they "saturate", to produce a vanishing entropy $\lim _{\sup _{n \rightarrow \infty}} \frac{1}{n} h_{n}^{A F}\left(\psi_{\hbar}, \mathcal{P}_{q}\right)$.

To prove the Theorem 2.1, we will still use the estimates (3.11), but in a more subtle way, namely by referring to an entropic uncertainty principle.

3.3. Entropic uncertainty principle. The theorem below is an adaptation of the entropic uncertainty principle conjectured by Deutsch and Kraus [12, 21] and proved by Massen and Uffink 25. These authors were investigating the theory of measurement in quantum mechanics. Roughly speaking, this result states that if a unitary matrix has "small" entries, then any of its eigenvectors must have a "large" Shannon entropy.

Let $(\mathcal{H},\langle.,\rangle$.$) be a complex Hilbert space, and denote \|\psi\|=\sqrt{\langle\psi, \psi\rangle}$ the associated norm. Consider a quantum partition of unity $\left(\pi_{k}\right)_{k=1}^{\mathcal{N}}$ on $\mathcal{H}$ as in (3.17). If $\|\psi\|=1$, we define the entropy of $\psi$ with respect to the partition $\pi$ as in (3.16) $)$, namely $h_{\pi}(\psi)=$ 
$\sum_{k=1}^{\mathcal{N}} \eta\left(\left\|\pi_{k} \psi\right\|^{2}\right)$. We extend this definition by introducing the notion of pressure, associated to a family $\boldsymbol{v}=\left(v_{k}\right)_{k=1, \ldots, \mathcal{N}}$ of positive real numbers: the pressure is defined by

$$
p_{\pi, \boldsymbol{v}}(\psi) \stackrel{\text { def }}{=} \sum_{k=1}^{\mathcal{N}} \eta\left(\left\|\pi_{k} \psi\right\|^{2}\right)-\sum_{k=1}^{\mathcal{N}}\left\|\pi_{k} \psi\right\|^{2} \log v_{k}^{2} .
$$

In Theorem [3.2, we actually need two partitions of unity $\left(\pi_{k}\right)_{k=1}^{\mathcal{N}}$ and $\left(\tau_{j}\right)_{j=1}^{\mathcal{M}}$, and two families of weights $\boldsymbol{v}=\left(v_{k}\right)_{k=1}^{\mathcal{N}}, \boldsymbol{w}=\left(w_{j}\right)_{j=1}^{\mathcal{M}}$, and consider the corresponding pressures $p_{\pi, \boldsymbol{v}}(\psi), p_{\tau, \boldsymbol{w}}(\psi)$. Besides the appearance of the weights $\boldsymbol{v}, \boldsymbol{w}$, we bring another modification to the statement in [25] by introducing an auxiliary operator $\mathcal{O}$.

Theorem 3.2. [4, Thm. 6.5] Let $\mathcal{O}$ be a bounded operator and $\mathcal{U}$ be an isometry on $\mathcal{H}$. Define $c_{\mathcal{O}}^{(\boldsymbol{v}, \boldsymbol{w})}(\mathcal{U}) \stackrel{\text { def }}{=} \sup _{j, k} w_{j} v_{k}\left\|\tau_{j} \mathcal{U} \pi_{k}^{*} \mathcal{O}\right\|$, and $V=\max _{k} v_{k}, W=\max _{j} w_{j}$.

Then, for any $\epsilon \geq 0$, for any normalized $\psi \in \mathcal{H}$ satisfying

$$
\forall k=1, \ldots, \mathcal{N}, \quad\left\|(I d-\mathcal{O}) \pi_{k} \psi\right\| \leq \epsilon,
$$

the pressures $p_{\tau, \boldsymbol{w}}(\mathcal{U} \psi), p_{\pi, \boldsymbol{v}}(\psi)$ satisfy

$$
p_{\tau, \boldsymbol{w}}(\mathcal{U} \psi)+p_{\pi, \boldsymbol{v}}(\psi) \geq-2 \log \left(c_{\mathcal{O}}^{(\boldsymbol{v}, \boldsymbol{w})}(\mathcal{U})+\mathcal{N} V W \epsilon\right) .
$$

Example 1. The original result of [25] corresponds to the case where $\mathcal{H}=\mathbb{C}^{\mathcal{N}}, \mathcal{O}=I d$, $\epsilon=0, \mathcal{N}=\mathcal{M}, v_{k}=w_{j}=1$, and the operators $\pi_{k}=\tau_{k}$ are the orthogonal projectors on some orthonormal basis $\left(e_{k}\right)_{k=1}^{\mathcal{N}}$ of $\mathcal{H}$. In this case, the theorem asserts that

$$
h_{\pi}(\mathcal{U} \psi)+h_{\pi}(\psi) \geq-2 \log c(\mathcal{U})
$$

where $c(\mathcal{U})=\sup _{j, k}\left|\left\langle e_{k}, \mathcal{U} e_{j}\right\rangle\right|$ is the supremum of all matrix elements of $\mathcal{U}$ in the orthonormal basis $\left(e_{k}\right)$. As a special case, one gets $h_{\pi}(\psi) \geq-\log c(\mathcal{U})$ if $\psi$ is an eigenfunction of $\mathcal{U}$.

3.4. Applying the entropic uncertainty principle to the Laplacian eigenstates. In this section we explain how to use Theorem 3.2 in order to obtain nontrivial information on the quantum entropies (3.16) and then $h_{K S}(\mu)$. For this we need to define the data to input in the theorem. Except the Hilbert space $\mathcal{H}=L^{2}(M)$, all other data depend on the semiclassical parameter $\hbar$ : the quantum partition $\pi$, the operator $\mathcal{O}$, the positive real number $\epsilon$, the weights $\left(v_{j}\right),\left(w_{k}\right)$ and the unitary operator $\mathcal{U}$.

As explained in section [3.2, we partition $M$ into $M=\sqcup_{k=1}^{K} M_{k}$, consider open sets $\Omega_{k} \supset M_{k}$ (which we assume to have diameters $\leq \varepsilon$ ), and consider smoothed characteristic functions $\mathbb{1}_{M_{k}}^{s m}$ supported respectively inside $\Omega_{k}$, and satisfying the identity (3.14). The associated multiplication operators on $\mathcal{H}$ are form a quantum partition $\left(P_{k}\right)_{k=1}^{K}$, which we had called $\mathcal{P}_{q}$. To alleviate notations, we will drop the subscript $q$.

From (3.15), and using the unitarity of $U_{\hbar}$, one realizes that for any $n \geq 1$, the families of operators $\mathcal{P}^{\vee n}=\left(P_{\boldsymbol{\alpha}}^{*}\right)_{|\boldsymbol{\alpha}|=n}$ and $\mathcal{T}^{\vee n}=\left(P_{\boldsymbol{\alpha}}\right)_{|\boldsymbol{\alpha}|=n}$ (see (3.10)) make up two quantum partitions of unity as in (3.17), of cardinal $K^{n}$. 
3.4.1. Sharp energy localization. In the estimate (3.11), we introduced an energy cutoff $\chi$ on a finite energy $\operatorname{strip} \mathcal{E}^{\varepsilon}$, with $\chi \equiv 1$ near $\mathcal{E}$. This cutoff does not appear in the estimate (3.9), because, when applied to the eigenstate $\psi_{\hbar}$, the operator $\mathrm{Op}_{\hbar}(\chi)$ essentially acts like the identity.

The estimate (3.11) will actually not suffice to prove Theorem 2.1. We will need to optimize it by replacing $\chi$ in (3.11) with a "sharp" energy cutoff. For some fixed (small) $\delta \in(0,1)$, we consider a smooth function $\chi_{\delta} \in C^{\infty}(\mathbb{R} ;[0,1])$, with $\chi_{\delta}(t)=1$ for $|t| \leq \mathrm{e}^{-\delta / 2}$ and $\chi_{\delta}(t)=0$ for $|t| \geq 1$. Then, we rescale that function to obtain the following family of $\hbar$-dependent cutoffs near $\mathcal{E}$ :

$$
\forall \hbar \in(0,1), \forall n \in \mathbb{N}, \forall \rho \in T^{*} M, \quad \chi^{(n)}(\rho ; \hbar) \stackrel{\text { def }}{=} \chi_{\delta}\left(\mathrm{e}^{-n \delta} \hbar^{-1+\delta}(H(\rho)-1 / 2)\right) .
$$

The cutoff $\chi^{(n)}$ is supported in a tubular neighbourhood of $\mathcal{E}$ of width $2 \hbar^{1-\delta} \mathrm{e}^{n \delta}$. We will always assume that this width is $<<\hbar^{1 / 2}$ in the semiclassical limit, which is the case if we ensure that $n \leq C_{\delta}|\log \hbar|$ for some $0<C_{\delta}<(2 \delta)^{-1}-1$. In spite of their singular behaviour, these cutoffs can be quantized into pseudodifferential operators $\operatorname{Op}\left(\chi^{(n)}\right)$ described in 4] (the quantization uses a pseudodifferential calculus adapted to the energy layer $\mathcal{E}$, drawn from [29]). The eigenstate $\psi_{\hbar}$ is indeed very localized near $\mathcal{E}$, since it satisfies

$$
\left\|\left(\operatorname{Op}\left(\chi^{(0)}\right)-1\right) \psi_{\hbar}\right\|=\mathcal{O}\left(\hbar^{\infty}\right)\left\|\psi_{\hbar}\right\| .
$$

In the rest of the paper, we also fix a small $\delta^{\prime}>0$, and call "Ehrenfest time" the $\hbar$-dependent integer

$$
n_{E}(\hbar) \stackrel{\text { def }}{=}\left\lfloor\frac{\left(1-\delta^{\prime}\right)|\log \hbar|}{\lambda_{\max }}\right\rfloor .
$$

Notice the resemblance with the time $n_{1}$ defined in (3.12). The significance of this time scale will be discussed in $\$ 3.4 .5$.

The following proposition states that the operators $\left(P_{\boldsymbol{\alpha}}^{*}\right)_{|\boldsymbol{\alpha}|=n_{E}}$, almost preserve the energy localization of $\psi_{\hbar}$ :

Proposition 3.3. For any $L>0$, there exists $\hbar_{L}$ such that, for any $\hbar \leq \hbar_{L}$, the Laplacian eigenstate satisfies

$$
\forall \boldsymbol{\alpha},|\boldsymbol{\alpha}|=n_{E}, \quad\left\|\left(\mathrm{Op}\left(\chi^{\left(n_{E}\right)}\right)-I d\right) P_{\boldsymbol{\alpha}}^{*} \psi_{\hbar}\right\| \leq \hbar^{L}\left\|\psi_{\hbar}\right\| .
$$

We recognize here a condition of the form (3.22).

3.4.2. Applying Theorem 3.2: Step 1. We now precise some of the data we will use in the entropic uncertainty principle, Theorem 3.2. As opposed to the choice made in [4], we will use two different partitions $\pi, \tau$.

- the quantum partitions $\pi$ and $\tau$ are given respectively by the families of operators $\pi=\mathcal{P}^{\vee n_{E}}=\left(P_{\boldsymbol{\alpha}}^{*}\right)_{|\boldsymbol{\alpha}|=n_{E}}, \tau=\mathcal{T}^{\vee n_{E}}=\left(P_{\boldsymbol{\alpha}}\right)_{|\boldsymbol{\alpha}|=n_{E}}$. Notice that these partitions only differ by the ordering of the operators $P_{\alpha_{i}}(i)$ inside the products. In the semiclassical limit, these partitions have cardinality $\mathcal{N}=K^{n_{E}} \asymp \hbar^{-K_{0}}$ for some fixed $K_{0}>0$.

- the isometry will be the propagator at the Ehrenfest time, $\mathcal{U}=U_{\hbar}^{n_{E}}$. 
- the auxiliarly operator is given as $\mathcal{O}=\operatorname{Op}\left(\chi^{\left(n_{E}\right)}\right)$, and the error $\epsilon=\hbar^{L}$, where $L$ will be chosen very large (see \$3.4.4).

- the weights $v_{\boldsymbol{\alpha}}, w_{\boldsymbol{\alpha}}$ will be selected in $\$ 3.4 .4$. They will be semiclassically tempered, meaning that there exists $K_{1}>0$ such that, for $\hbar$ small enough, all $v_{\boldsymbol{\alpha}}, w_{\boldsymbol{\alpha}}$ are contained in the interval $\left[1, \hbar^{-K_{1}}\right]$.

The entropy and pressures associated with a state $\psi \in \mathcal{H}$ are given by

$$
\begin{aligned}
h_{\pi}(\psi) & =\sum_{|\boldsymbol{\alpha}|=n_{E}} \eta\left(\left\|P_{\boldsymbol{\alpha}}^{*} \psi\right\|^{2}\right), \\
p_{\pi, \boldsymbol{v}}(\psi) & =h_{\pi}(\psi)-2 \sum_{|\boldsymbol{\alpha}|=n_{E}}\left\|P_{\boldsymbol{\alpha}}^{*} \psi\right\|^{2} \log v_{\boldsymbol{\alpha}} .
\end{aligned}
$$

With respect to the second partition, we have

$$
\begin{aligned}
h_{\tau}(\psi) & =\sum_{|\boldsymbol{\alpha}|=n_{E}} \eta\left(\left\|P_{\boldsymbol{\alpha}} \psi\right\|^{2}\right), \\
p_{\tau, \boldsymbol{w}}(\psi) & =h_{\tau}(\psi)-2 \sum_{|\boldsymbol{\alpha}|=n_{E}}\left\|P_{\boldsymbol{\alpha}} \psi\right\|^{2} \log w_{\boldsymbol{\alpha}} .
\end{aligned}
$$

We notice that the entropy $h_{\tau}(\psi)$ exactly corresponds to the formula (3.16)), while $h_{\pi}(\psi)$ is built from the norms

$$
\left\|P_{\boldsymbol{\alpha}}^{*} \psi\right\|^{2}=\left\|P_{\alpha_{0}} P_{\alpha_{1}}(1) \cdots P_{\alpha_{n-1}}(n-1) \psi\right\|^{2} .
$$

If $\psi$ is an eigenfunction of $U_{\hbar}$, the above norm can be obtained from (3.13) by exchanging $U_{\hbar}$ with $U_{\hbar}^{-1}$, and replacing the sequence $\boldsymbol{\alpha}=\left(\alpha_{0}, \ldots, \alpha_{n-1}\right)$ by $\overline{\boldsymbol{\alpha}} \stackrel{\text { def }}{=}\left(\alpha_{n-1}, \ldots, \alpha_{0}\right)$. So the entropies $h_{\pi}(\psi)$ and $h_{\tau}(\psi)$ are mapped to one another through the time reversal $U_{\hbar} \rightarrow U_{\hbar}^{-1}$.

With these data, we draw from Theorem 3.2 the following

Corollary 3.4. For $\hbar>0$ small enough consider the data $\pi, \tau, \mathcal{U}, \mathcal{O}$ as defined above. Let

$$
c_{\mathcal{O}}^{\boldsymbol{v}, \boldsymbol{w}}(\mathcal{U}) \stackrel{\text { def }}{=} \max _{|\boldsymbol{\alpha}|=\left|\boldsymbol{\alpha}^{\prime}\right|=n_{E}}\left(w_{\boldsymbol{\alpha}^{\prime}} v_{\boldsymbol{\alpha}}\left\|P_{\boldsymbol{\alpha}^{\prime}} U_{\hbar}^{n_{E}} P_{\boldsymbol{\alpha}} \mathrm{Op}\left(\chi^{\left(n_{E}\right)}\right)\right\|\right)
$$

Then for any normalized state $\phi$ satisfying (3.26),

$$
p_{\tau, \boldsymbol{w}}\left(U_{\hbar}^{n_{E}} \phi\right)+p_{\pi, \boldsymbol{v}}(\phi) \geq-2 \log \left(c_{\mathcal{O}}^{\boldsymbol{v}, \boldsymbol{w}}(\mathcal{U})+h^{L-K_{0}-2 K_{1}}\right) .
$$

From (3.26), we see that the above corollary applies to the eigenstate $\psi_{\hbar}$ if $\hbar$ is small enough.

The reason to take the same value $n_{E}$ for the refined partitions $\mathcal{P}^{\vee n_{E}}, \mathcal{T}^{\vee n_{E}}$ and the propagator $U_{\hbar}^{n_{E}}$ is the following : the products appearing in $c_{\mathcal{O}}^{\boldsymbol{v}, \boldsymbol{w}}(\mathcal{U})$ can be rewritten (with $U \equiv U_{\hbar}$ ):

$$
P_{\boldsymbol{\alpha}^{\prime}} U^{n_{E}} P_{\boldsymbol{\alpha}}=U^{-n_{E}+1} P_{\alpha_{n_{E}-1}^{\prime}} U \cdots U P_{\alpha_{0}^{\prime}} U P_{\alpha_{n_{E}}-1} U \cdots U P_{\alpha_{0}}=U^{n_{E}} P_{\boldsymbol{\alpha} \boldsymbol{\alpha}^{\prime}}
$$


Thus, the estimate (3.11) with $n=2 n_{E}$ already provides an upper bound for the norms appearing in (3.31) - the replacement of $\chi$ by the sharp cutoff $\chi^{\left(n_{E}\right)}$ does not harm the estimate.

To prove Theorem 2.1, we actually need to improve the estimate (3.11), as was done in [4], see Theorem 3.5 below. This improvement is done at two levels: we will use the fact that the cutoffs $\chi^{\left(n_{E}\right)}$ are sharper than $\chi$, and also the fact that the expansion rate of the geodesic flow (which governs the upper bound in (3.11)) is not uniform, but depends on the sequence $\boldsymbol{\alpha}$.

Our choice for the weights $v_{\boldsymbol{\alpha}}, w_{\boldsymbol{\alpha}}$ will then be guided by the $\boldsymbol{\alpha}$-dependent upper bounds given in Theorem 3.5. To state that theorem, we introduce some notations.

3.4.3. Coarse-grained unstable Jacobian. We recall that, for any energy $\lambda>0$, the geodesic flow $g^{t}$ on the energy layer $\mathcal{E}(\lambda)=H^{-1}(\lambda) \subset T^{*} M$ is Anosov, so that the tangent space $T_{\rho} \mathcal{E}(\lambda)$ at each $\rho \in T^{*} M, H(\rho)>0$ splits into

$$
T_{\rho} \mathcal{E}(\lambda)=E^{u}(\rho) \oplus E^{s}(\rho) \oplus \mathbb{R} X_{H}(\rho)
$$

where $E^{u}$ (resp. $\left.E^{s}\right)$ is the unstable (resp. stable) subspace. The unstable Jacobian $J^{u}(\rho)$ is defined by $J^{u}(\rho)=\operatorname{det}\left(d g_{\mid E^{u}\left(g^{1} \rho\right)}^{-1}\right)$ (the unstable spaces at $\rho$ and $g^{1} \rho$ are equipped with the induced Riemannian metric).

This Jacobian can be "discretized" as follows in the energy strip $\mathcal{E}^{\varepsilon} \supset \mathcal{E}$. For any pair of indices $\left(\alpha_{0}, \alpha_{1}\right) \in \llbracket 1, K \rrbracket^{2}$, we define

$$
J_{1}^{u}\left(\alpha_{0}, \alpha_{1}\right) \stackrel{\text { def }}{=} \sup \left\{J^{u}(\rho): \rho \in T^{*} \Omega_{\alpha_{0}} \cap \mathcal{E}^{\varepsilon}, g^{1} \rho \in T^{*} \Omega_{\alpha_{1}}\right\}
$$

if the set on the right hand side is not empty, and $J_{1}^{u}\left(\alpha_{0}, \alpha_{1}\right)=e^{-R}$ otherwise, where $R>0$ is a fixed large number. For any sequence of symbols $\boldsymbol{\alpha}$ of length $n$, we define

$$
J_{n}^{u}(\boldsymbol{\alpha}) \stackrel{\text { def }}{=} J_{1}^{u}\left(\alpha_{0}, \alpha_{1}\right) \cdots J_{1}^{u}\left(\alpha_{n-2}, \alpha_{n-1}\right) .
$$

Although $J^{u}$ and $J_{1}^{u}\left(\alpha_{0}, \alpha_{1}\right)$ are not necessarily everywhere smaller than unity, there exists $C, \lambda_{+}, \lambda_{-}>0$ such that, for any $n>0$, for any $\boldsymbol{\alpha}$ with $|\boldsymbol{\alpha}|=n$,

$$
C^{-1} \mathrm{e}^{-n(d-1) \lambda_{+}} \leq J_{n}^{u}(\boldsymbol{\alpha}) \leq C \mathrm{e}^{-n(d-1) \lambda_{-}} .
$$

One can take $\lambda_{+}=\lambda_{\max }(1+\varepsilon)$, where $\lambda_{\max }$ is the maximal expanding rate in Theorem. 2.1, We now give our central estimate, easy to draw from [4, Corollary 3.4].

Theorem 3.5. Fix small positive constants $\varepsilon, \delta$, $\delta^{\prime}$ and a constant $0<C_{\delta}<(2 \delta)^{-1}-1$. Take an open cover $M=\bigcup_{k} \Omega_{k}$ of diameter $\leq \varepsilon$ and an associated quantum partition $\mathcal{P}=$ $\left(P_{k}\right)_{k=1}^{K}$. There exists $\hbar_{0}$ such that, for any $\hbar \leq \hbar_{0}$, for any positive integer $n \leq C_{\delta}|\log \hbar|$, and any pair of sequences $\boldsymbol{\alpha}, \boldsymbol{\alpha}^{\prime}$ of length $n$,

$$
\left\|P_{\boldsymbol{\alpha} \boldsymbol{\alpha}^{\prime}} \mathrm{Op}\left(\chi^{(n)}\right)\right\|=\left\|P_{\boldsymbol{\alpha}^{\prime}} U_{\hbar}^{n} P_{\boldsymbol{\alpha}} \mathrm{Op}\left(\chi^{(n)}\right)\right\| \leq C \hbar^{-\frac{d-1}{2}-\delta} \mathrm{e}^{n \delta} \sqrt{J_{n}^{u}(\boldsymbol{\alpha}) J_{n}^{u}\left(\boldsymbol{\alpha}^{\prime}\right)} .
$$

The constant $C$ only depends on the Riemannian manifold $(M, g)$. If we take $n=n_{E}$, this takes the form

$$
\left\|P_{\boldsymbol{\alpha}^{\prime}} U_{\hbar}^{n_{E}} P_{\boldsymbol{\alpha}} \mathrm{Op}\left(\chi^{\left(n_{E}\right)}\right)\right\| \leq C \hbar^{-\frac{d-1+c \delta}{2}} \sqrt{J_{n_{E}}^{u}(\boldsymbol{\alpha}) J_{n_{E}}^{u}\left(\boldsymbol{\alpha}^{\prime}\right)}
$$


where $c=2+2 \lambda_{\max }^{-1}$.

The idea of proof in Theorem 3.5 is rather simple, although the technical implementation is cumbersome. We first show that for any normalized state $\psi$, the state $\operatorname{Op}\left(\chi^{(n)}\right) \psi$ can be essentially decomposed into a superposition of $\hbar^{-d}\left|\operatorname{supp} \chi^{(n)}\right|$ normalized Lagrangian states, supported on Lagrangian manifolds transverse to the stable foliation. In fact the Lagrangian states we work with are truncated $\delta$-functions, supported on lagrangians of the form $\cup_{t} g^{t} S_{z}^{*} M$. The action of the operator $U^{n} P_{\boldsymbol{\alpha} \alpha^{\prime}}=P_{\alpha_{n-1}^{\prime}} U \cdots U P_{\alpha_{0}}$ on such Lagrangian states can be analyzed through WKB methods, and is simple to understand at the classical level : each application of the propagator $U$ stretches the Lagrangian along the unstable direction (the rate of stretching being described by the local unstable Jacobian), whereas each operator $P_{k}$ "projects" on a piece of Lagrangian of diameter $\varepsilon$. This iteration of stretching and cutting accounts for the exponential decay. The $\boldsymbol{\alpha} \boldsymbol{\alpha}^{\prime}$-independent factor on the right of (3.36) results from adding together the contributions of all the initial Lagrangian states. Notice that this prefactor is smaller than in Theorem. 3.1 due to the condition $C_{\delta}<(2 \delta)^{-1}-1$.

Remark 3.6. In [4] we used the same quantum partition $\mathcal{P}^{\vee n_{E}}$ for $\pi$ and $\tau$ in Theorem. 3.2. As a result, we needed to estimate from above the norms $\left\|P_{\boldsymbol{\alpha}^{\prime}}^{*} U^{n_{E}} P_{\boldsymbol{\alpha}} \mathrm{Op}\left(\chi^{\left(n_{E}\right)}\right)\right\|$ (see [4, Theorem. 2.6]). The proof of this estimate was much more involved than the one for (3.36), since it required to control long pieces of unstable manifolds. By using instead the two partitions $\mathcal{P}^{(n)}, \mathcal{T}^{(n)}$, we not only prove a more precise lower bound (2.3) on the KS entropy, but also short-circuit some fine dynamical analysis.

3.4.4. Applying Theorem 3.2: Step 2. There remains to choose the weights $\left(v_{\boldsymbol{\alpha}}, w_{\boldsymbol{\alpha}}\right)$ to use in Theorem 3.2. Our choice is guided by the following idea: in (3.31), the weights should balance the variations (with respect to $\boldsymbol{\alpha}, \boldsymbol{\alpha}^{\prime}$ ) in the norms, such as to make all terms in (3.31) of the same order. Using the upper bounds (3.36), we end up with the following choice for all $\boldsymbol{\alpha}$ of length $n_{E}$ :

$$
v_{\boldsymbol{\alpha}}=w_{\boldsymbol{\alpha}} \stackrel{\text { def }}{=} J_{n_{E}}^{u}(\boldsymbol{\alpha})^{-1 / 2} .
$$

From (3.34), there exists $K_{1}>0$ such that, for $\hbar$ small enough, all the weights are contained in the interval $\left[1, \hbar^{-K_{1}}\right]$, as announced in $\$ 3.4 .2$. Using these weights, the estimate (3.36) implies the following bound on the coefficient (3.31):

$$
\forall \hbar<\hbar_{0}, \quad c_{\mathcal{O}}^{\boldsymbol{v}, \boldsymbol{w}}(\mathcal{U}) \leq C \hbar^{-\frac{d-1+c \delta}{2}} .
$$

We can now apply Corollary 3.4 to the particular case of the eigenstates $\psi_{\hbar}$. We choose $L$ such that $L-K_{0}-2 K_{1}>-\frac{d-1+c \delta}{2}$, so from Corollary 3.4 we draw the following

Proposition 3.7. Let $\left(\psi_{\hbar}\right)_{\hbar \rightarrow 0}$ be our sequence of eigenstates (2.2). In the semiclassical limit, the pressures of $\psi_{\hbar}$ satisfy

$$
p_{\mathcal{P}^{\vee n_{E}, \boldsymbol{v}}}\left(\psi_{\hbar}\right)+p_{\mathcal{T}^{\vee n_{E}, \boldsymbol{w}}}\left(\psi_{\hbar}\right) \geq-\frac{(d-1+c \delta) \lambda_{\max }}{\left(1-\delta^{\prime}\right)} n_{E}+\mathcal{O}(1) .
$$


If $M$ has constant curvature we have $\log J_{\boldsymbol{\alpha}}^{n} \leq-n(d-1) \lambda_{\max }(1-\mathcal{O}(\varepsilon))$ for all $\boldsymbol{\alpha}$ of length $n$, and the above lower bound can be written

$$
h_{\mathcal{P}^{\vee n_{E}}}\left(\psi_{\hbar}\right)+h_{\mathcal{T}^{\vee n_{E}}}\left(\psi_{\hbar}\right) \geq(d-1) \lambda_{\max }\left(1+\mathcal{O}\left(\varepsilon, \delta, \delta^{\prime}\right)\right) n_{E} .
$$

As opposed to (3.21), the above inequality provides a nontrivial lower bound for the quantum entropies at the time $n_{E}$, which is smaller than the time $n_{1}$ of (3.12), and will allow to connect those entropies to the KS entropy of the semiclassical measure (see below).

3.4.5. Subadditivity until the Ehrenfest time. Even at the relatively small time $n_{E}$, the connection between the quantum entropy $h\left(\psi_{\hbar}, \mathcal{P}^{\vee n_{E}}\right)$ and the classical $h\left(\mu, \mathcal{P}_{s m}^{\vee n_{E}}\right)$ is not completely obvious: both are sums of a large number of terms $\left(\asymp \hbar^{-K_{0}}\right)$. Before taking the limit $\hbar \rightarrow 0$, we will prove that a lower bound of the form (3.37) still holds if we replace $n_{E} \asymp|\log \hbar|$ by some fixed $n_{o} \in \mathbb{N}$, and $\mathcal{P}^{\vee n_{E}}$ by the corresponding quantum partition $\mathcal{P}^{\vee n_{o}}$. The link between quantum pressures at times $n_{E}$ and $n_{o}$ is provided by the following subadditivity property, which is the semiclassical analogue of the classical subadditivity of pressures for invariant measures (see (3.3.3) ).

Proposition 3.8 (Subadditivity). Let $\delta^{\prime}>0$. There is a function $R\left(n_{o}, \hbar\right)$, and a real number $R>0$ independent of $\delta^{\prime}$, such that, for any integer $n_{o} \geq 1$,

$$
\limsup _{\hbar \longrightarrow 0}\left|R\left(n_{o}, \hbar\right)\right| \leq R
$$

and with the following properties. For any small enough $\hbar>0$, any integers $n_{o}, n \in \mathbb{N}$ with $n_{o}+n \leq n_{E}(\hbar)$, for any $\psi_{\hbar}$ normalized eigenstate satisfying (2.2), the following inequality holds:

$$
p_{\mathcal{P} \vee\left(n_{o}+n\right), v}\left(\psi_{\hbar}\right) \leq p_{\mathcal{P} \vee n_{o}, \boldsymbol{v}}\left(\psi_{\hbar}\right)+p_{\mathcal{P} \vee n, v}\left(\psi_{\hbar}\right)+R\left(n_{o}, \hbar\right) .
$$

The same inequality is satisfied by the pressures $p_{\mathcal{T}^{\vee n}, \boldsymbol{w}}\left(\psi_{\hbar}\right)$.

To prove this proposition, one uses a refined version of Egorov's theorem [10] to show that the non-commutative dynamical system formed by $\left(U_{\hbar}^{t}\right)$ acting (through Heisenberg) on observables supported near $\mathcal{E}$ is (approximately) commutative on time intervals of length $n_{E}(\hbar)$. Precisely, we showed in [4] that, provided $\varepsilon$ is small enough, for any $a, b \in C_{c}^{\infty}\left(\mathcal{E}^{\varepsilon}\right)$,

$$
\forall t \in\left[-n_{E}(\hbar), n_{E}(\hbar)\right], \quad\left\|\left[\mathrm{Op}_{\hbar}(a)(t), \mathrm{Op}_{\hbar}(b)\right]\right\|=\mathcal{O}\left(\hbar^{c \delta^{\prime}}\right), \quad \hbar \rightarrow 0,
$$

and the implied constant is uniform with respect to $t$. Within that time interval, the operators $P_{\alpha_{j}}(j)$ appearing in the definition of the pressures commute up to small semiclassical errors. This almost commutativity explains why the quantum pressures $p_{\mathcal{P}^{\vee n}, \boldsymbol{v}}\left(\psi_{\hbar}\right)$ satisfy the same subadditivity property as the classical entropy (3.3), for times smaller than $n_{E}$.

Thanks to this subadditivity, we may finish the proof of Theorem. 2.1. Fixing $n_{o}$, using for each $\hbar$ the Euclidean division $n_{E}(\hbar)=q(\hbar) n_{o}+r(\hbar)$ (with $r(\hbar)<n_{o}$ ), Proposition 3.8 implies that for $\hbar$ small enough,

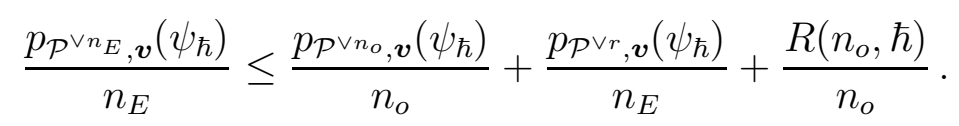


The same inequality is satisfied by the pressures $p_{\mathcal{T}^{\vee n}, \boldsymbol{w}}\left(\psi_{\hbar}\right)$. Using (3.37) and the fact that $p_{\mathcal{P}}^{\vee r}, \boldsymbol{v}\left(\psi_{\hbar}\right)$ stays uniformly bounded when $\hbar \rightarrow 0$, we find

$$
\frac{p_{\mathcal{P}^{\vee n_{o}, \boldsymbol{v}}}\left(\psi_{\hbar}\right)+p_{\mathcal{T}^{\vee n_{o}, \boldsymbol{w}}}\left(\psi_{\hbar}\right)}{n_{o}} \geq-\frac{2(d-1+c \delta) \lambda_{\max }}{2\left(1-\delta^{\prime}\right)}-\frac{2 R\left(n_{o}, \hbar\right)}{n_{o}}+\mathcal{O}_{n_{o}}\left(1 / n_{E}\right) .
$$

We are now dealing with quantum partitions $\mathcal{P}^{\vee n_{o}}, \mathcal{T}^{\vee n_{o}}$, for $n_{0} \in \mathbb{N}$ independent of $\hbar$. At this level the quantum and classical entropies are related through the (finite time) Egorov theorem, as we had noticed in $\$ 3.2 .2$. For any $\boldsymbol{\alpha}$ of length $n_{o}$, the weights $\left\|P_{\boldsymbol{\alpha}} \psi_{\hbar}\right\|^{2}$ and $\left\|P_{\boldsymbol{\alpha}}^{*} \psi_{\hbar}\right\|^{2}$ both converge to $\mu\left(\left(\mathbb{1}_{M_{\boldsymbol{\alpha}}}^{s m}\right)^{2}\right)$, where we recall that

$$
\mathbb{1}_{M_{\alpha}}^{s m}=\left(\mathbb{1}_{M_{\alpha_{n_{0}-1}}}^{s m} \circ g^{n_{o}-1}\right) \times \ldots \times\left(\mathbb{1}_{M_{\alpha_{1}}}^{s m} \circ g\right) \times \mathbb{1}_{M_{\alpha_{0}}}^{s m} .
$$

Thus, both entropies $h_{\mathcal{P} \vee n_{o}}\left(\psi_{\hbar}\right), h_{\mathcal{T} \vee n_{o}}\left(\psi_{\hbar}\right)$ semiclassically converge to the classical entropy $h_{n_{o}}\left(\mu, \mathcal{P}_{s m}\right)$. As a result, the left hand side of (3.38) converges to

$$
2 \frac{h_{n_{o}}\left(\mu, \mathcal{P}_{s m}\right)}{n_{o}}+\frac{2}{n_{o}} \sum_{|\boldsymbol{\alpha}|=n_{o}} \mu\left(\left(\mathbb{1}_{M_{\boldsymbol{\alpha}}}^{s m}\right)^{2}\right) \log J_{n_{o}}^{u}(\boldsymbol{\alpha}) .
$$

Since $\mu$ is $g^{t}$-invariant and $J_{n_{o}}^{u}$ has the multiplicative structure (3.33), the second term in (3.39) can be simplified:

$$
\sum_{|\boldsymbol{\alpha}|=n_{o}} \mu\left(\left(\mathbb{1}_{M_{\boldsymbol{\alpha}}}^{s m}\right)^{2}\right) \log J_{n_{o}}^{u}(\boldsymbol{\alpha})=\left(n_{o}-1\right) \sum_{\alpha_{0}, \alpha_{1}} \mu\left(\left(\mathbb{1}_{M_{\left(\alpha_{0}, \alpha_{1}\right)}}^{s m}\right)^{2}\right) \log J_{1}^{u}\left(\alpha_{0}, \alpha_{1}\right) .
$$

We have thus obtained the lower bound

$$
\frac{h_{n_{o}}\left(\mu, \mathcal{P}_{s m}\right)}{n_{o}} \geq-\frac{n_{o}-1}{n_{o}} \sum_{\alpha_{0}, \alpha_{1}} \mu\left(\left(\mathbb{1}_{M_{\left(\alpha_{0}, \alpha_{1}\right)}^{s m}}\right)^{2}\right) \log J_{1}^{u}\left(\alpha_{0}, \alpha_{1}\right)-\frac{(d-1+c \delta) \lambda_{\max }}{2\left(1-\delta^{\prime}\right)}-\frac{R}{n_{o}}
$$

At this stage we may forget about $\delta$ and $\delta^{\prime}$. The above lower bound does not depend on the derivatives of the functions $\mathbb{1}_{M_{\alpha}}^{s m}$, so the same bound carries over if we replace $\mathbb{1}_{M_{\alpha}}^{s m}$ by the characteristic functions $\mathbb{1}_{M_{\alpha}}$. We can finally let $n_{o}$ tend to $+\infty$, then let the diameter $\varepsilon$ tend to 0 . The left hand side converges to $h_{K S}(\mu)$ while, from the definition (3.32), the sum in the right hand side of (3.40) converges to the integral $\int_{\mathcal{E}} \log J^{u}(\rho) d \mu(\rho)$ as $\varepsilon \rightarrow 0$, which proves (2.3).

\section{REFERENCES}

[1] R. Alicki and M. Fannes, Defining quantum dynamical entropy, Lett. Math. Phys. 32 75-82 (1994)

[2] N. Anantharaman, Entropy and the localization of eigenfunctions, to appear in Ann. Math.

[3] N. Anantharaman and S. Nonnenmacher, Entropy of semiclassical measures of the Walshquantized baker's map, Ann. H. Poincaré 8, 37-74 (2007)

[4] N. Anantharaman, S. Nonnenmacher, Half-delocalization of eigenfunctions of the laplacian on an Anosov manifold, http://hal.archives-ouvertes.fr/hal-00104963

[5] F. Benatti, V. Cappellini, M. De Cock, M. Fannes and D. Van Peteghem, Classical Limit of Quantum Dynamical Entropies, Rev. Math. Phys. 15, 1-29 (2003)

[6] M.V. Berry, Regular and irregular semiclassical wave functions, J.Phys. A 10, 2083-2091 (1977) 
[7] O. Bohigas, Random matrix theory and chaotic dynamics, in M.J. Giannoni, A. Voros and J. ZinnJustin eds., Chaos et physique quantique, (École d'été des Houches, Session LII, 1989), North Holland, 1991

[8] J. Bourgain, E. Lindenstrauss, Entropy of quantum limits, Comm. Math. Phys. 233, 153-171 (2003).

[9] A. Bouzouina and S. De Bièvre, Equipartition of the eigenfunctions of quantized ergodic maps on the torus, Commun. Math. Phys. 178 (1996) 83-105

[10] A. Bouzouina and D. Robert: Uniform Semi-classical Estimates for the Propagation of Quantum Observables, Duke Math. J. 111, 223-252 (2002)

[11] Y. Colin de Verdière, Ergodicité et fonctions propres du laplacien, Commun. Math. Phys. 102, 497-502 (1985)

[12] D. Deutsch, Uncertainty in quantum measurements, Phys. Rev. Lett. 50, 631-633 (1983)

[13] M. Dimassi, J. Sjöstrand, Spectral asymptotics in the semi-classical limit, London Mathematical Society Lecture Note Series, 268. Cambridge University Press, Cambridge, 1999.

[14] L.C. Evans and M. Zworski, Lectures on semiclassical analysis (version 0.2), available at http://math. berkeley.edu/ zworski

[15] F. Faure, S. Nonnenmacher and S. De Bièvre, Scarred eigenstates for quantum cat maps of minimal periods, Commun. Math. Phys. 239, 449-492 (2003).

[16] F. Faure and S. Nonnenmacher, On the maximal scarring for quantum cat map eigenstates, Commun. Math. Phys. 245, 201-214 (2004)

[17] R.B. Griffiths, Consistent Histories and the interpretation of quantum mechanics, J. Stat. Phys. 36, 219-272 (1984)

[18] J.H. Hannay and M.V. Berry, Quantisation of linear maps on the torus-Fresnel diffraction by a periodic grating, Physica D 1, 267-290 (1980)

[19] A. Katok and B. Hasselblatt, Introduction to the modern theory of dynamical systems, Encyclopedia of Mathematics and its applications vol.54, Cambridge University Press, 1995.

[20] D. Kelmer, Arithmetic quantum unique ergodicity for symplectic linear maps of the multidimensional torus, preprint (2005) math-ph/0510079

[21] K. Kraus, Complementary observables and uncertainty relations, Phys. Rev. D 35, 3070-3075 (1987)

[22] P. Kurlberg and Z. Rudnick, Hecke theory and equidistribution for the quantization of linear maps of the torus, Duke Math. J. 103, 47-77 (2000)

[23] F. Ledrappier, L.-S. Young, The metric entropy of diffeomorphisms. I. Characterization of measures satisfying Pesin's entropy formula, Ann. of Math. (2) 122 (1985), no. 3, 509-539.

[24] E. Lindenstrauss, Invariant measures and arithmetic quantum unique ergodicity, Annals of Math. 163, 165-219 (2006)

[25] H. Maassen and J.B.M. Uffink, Generalized entropic uncertainty relations, Phys. Rev. Lett. 60, 1103-1106 (1988)

[26] Z. Rudnick and P. Sarnak, The behaviour of eigenstates of arithmetic hyperbolic manifolds, Commun. Math. Phys. 161, 195-213 (1994)

[27] A. Schnirelman, Ergodic properties of eigenfunctions, Usp. Math. Nauk. 29, 181-182 (1974)

[28] W. Słomczyński and K. Życzkowski, Quantum chaos: an entropy approach, J. Math. Phys. 35, $5674-5700$ (1994)

[29] J. Sjöstrand and M. Zworski, Asymptotic distribution of resonances for convex obstacles, Acta Math. 183, 191-253 (1999)

[30] A. Voros, Semiclassical ergodicity of quantum eigenstates in the Wigner representation, Lect. Notes Phys. 93, 326-333 (1979) in: Stochastic Behavior in Classical and Quantum Hamiltonian Systems, G. Casati, J. Ford, eds., Proceedings of the Volta Memorial Conference, Como, Italy, 1977, Springer, Berlin 
[31] S.A. Wolpert, The modulus of continuity for $\Gamma_{0}(m) / \mathbb{H}$ semi-classical limits, Commun. Math. Phys. 216, 313-323 (2001)

[32] S. Zelditch, Uniform distribution of the eigenfunctions on compact hyperbolic surfaces, Duke Math. J. 55, 919-941 (1987)

CMls, École Polytechnique, 91128 Palaiseau, France

E-mail address: nalini@math.polytechnique.fr

Mathematical Institute, University of Bonn, Beringstrasse 1,D-53115 Bonn, Germany

E-mail address: koch@math.uni-bonn.de

Service de Physique Théorique, CEA/DSM/Pht, Unité de Recherche associée au CNRS, CEA/Saclay, 91191 Gif-Sur-Yvette, France

E-mail address: snonnenmacher@cea.fr 